cloud, and noticed that it had what I may compare to a bowstring stretched from end to end. On Thursday, July s 9 , from II to 12 p.m., the whole sky was divided by such band; converging east and west. This was noticed by many persons in Essex, where I was staying.

3I, Meadow Road, S.W.

\section{ON MOUNTING AND PHOTOGRAPHING MICROSCOPIC OBJECTS ${ }^{1}$}

11.

THE prepared slide fixed in a clip should be placed on a hob or in a cool oven (not above $50^{\circ} \mathrm{C}$.) for two days, by which time the excess of balsam round the edge of the cover will have become brittle, and can be removed with the point of a scalpel or penknife. Any balsam still remaining can be cleaned off with methylated spirit and a clean soft rag. The final cleaning of the slide may be done with soap and water. As the balsam itself serves to secure the cover to the slide, no cement or varnish is needed, and it remains only to label the object.

After successfully mounting this object, no difficulty will be experienced in applying the same methods to other small insects and parts of insects, such as antennæ, spiracles, feet, wings, ovipositors, corneas, tracheæ, \&c. The two last cases, however, require careful dissection.

Animal hairs are best mounted in balsam, and the only special treatment they require is soaking for a short time in ether to remove grease.

The siliceous skeletons of diatoms and spiculæ of sponges and Holothuriæ require cleaning from extraneous matter by treatment with strong acids, but space will not allow a description of the details of their preparation.

The mounting of the organs and tissues of the higher animals and plants should not be attempted until tolerable facility has been acquired in the preparation of the simpler objects previously mentioned, as their structure is usually revealed only by the somewhat difficult process of cutting thin sections of them.

Most animal substances require hardening before they can be cut. Hardening may be thus effected. The perfectly fresh tissue is to be cut into pieces about the size of Spanish nuts, and soaked in ten times its bulk of a solution, consisting of one part of methylated spirit, and two parts of a $\frac{1}{2}$ per cent. solution of chromic acid. A the end of twenty-four hours, and again after every third day, the solution is to be changed. After a week or fortnight the pieces should be well washed in methylated spirit, and will then be hard enough for cutting.

The next process is to embed the tissue in some substance firm enough to afford it support, yet soft enough to be readily cut with it. A good material for this purpose is a mixture of three or four parts of solid paraffin (paraffin candles), three of lard, and one of paraffin oil. It should be heated just sufficiently to keep it fluid, and the hardened tissue from which the exc ss of alcohol has been drained should be soaked in it for a quarter of an hour if of moderately close texture. If of very open texture-lung or testis, for instance-it must be soaked for about half an lour in rectified alcohol, and for a like period in absolute alcobol, to remove all traces of water. Then after displacing the alcohol by a quarter or half an hour's immersion in oil of turpentine, the tissue may be placed in the melted wax, which being readily miscible with the turpentine, will gain access to all the interstices of the substance.

A mould must then be prepared by gumming a piece of paper round a cork or cylinder of wood, the paper being allowed to project about three-quarters of an inch. Into this mould the substance is to be put, and the space filled up with some of the melted wax. When quite cold the paper may be stripped off, and the preparation will be

I Concluded from p. 303. ready for cutting with a razor, wetted with spirit to prevent adhesion of the sections.

The sections as they are cut are to be floated off the razor into methylated spirit, from which they may be transferred to a staining fluid.

The object of staining is in most cases not simply to impart a general colour to the object, but to take advantage of the fact that different parts are affected in different degrees by the same dye and are thereby clearly discriminated. Thus if an ammoniacal solution of carmine be employed, the structures which are first and most deeply stained are nuclei, axis cylinders of nerves, and ganglion corpuscles. To a less extent it stains the protoplasm of gland-cells and connective tissue corpuscles. But if the action be too long continued, the whole will be deeply and uniformly stained, and the selective power will be lost.

Carmine solution may be prepared by dissolving with the aid of gentle heat 2 grammes of carmine in 4 c.c. of ammonia and 48 c.c. of distilled water. Continue the heat or expose to the air until the smell of ammonia has almost disappeared, and then keep in a well-corked bottle. When required for use, a few drops of this solution should be added to a watch-glass full of water.

Logwood resembles carmine in its action and is by many preferred to it. It may be prepared as follows :I2 grammes of extract of logwood and 36 grammes of alum, both in fine powder, are to be mixed with 60 c.c. of distilled water, stirred well with a glass rod and filtered. Add to filtrate 5 c.c. rectified alcohol. Dilute with two or three times its volume of distilled water when used. When the tissue has been hardened with chromic acid, the sections should be soaked for a few minutes in a I per cent. solution of sodic bicarbonate to neutralise the acid before staining in logwood.

No general rule can be given for the length of time the section must remain in the staining fluid. It will vary from a few minutes to as many hours, and the section must be removed and examined with the microscope from time to time to see when the process has gone far enough.

When sufficiently stained, the excess of staining fluid is to be drained off and the section passed through rectified spirit 60 O.P., oil of cloves, and oil of turpentine, remaining about five minutes in each, and may then be mounted in balsam as already described.

For displaying tesselated epithelium in mesenteries, lungs, and blood-vessels, nothing can be more beautiful than staining by oxide of silver reduced from the nitrate. The perfectly fresh membrane or the section of hardened tissue as the case may be must be well washed with distilled water and then soaked for five minutes in a 5 per cent. solution of nitrate of silver. It is then again to be washed and exposed in distilled water to sunlight until it assumes a brown colour. The necessary exposure will vary from ten minutes to an hour or more. After a final wash in distilled water, it may be treated like objects stained by other methods. By this treatment the tissue assumes a general pale brown tint and the outline of every cell is sharply marked out by a deep brown deposit of argentic oxide in the intercellular substance.

Many vegetable tissues, such as cork, pith, succulent leaves, and some fruits, tubers, and roots, can be cut without previous preparation, and for such as are too soft to be cut in the fresh state the process of hardening is simpler than that employed for animal substances. Dehydration by simply soaking for a day or two in methylated spirit usually suffices.

Stems of plants usually require softening before cutting, and this softening can be effected if the wood is young by two or three days' immersion in methylated spirit to remove resinous matter, followed by maceration for from four days to a week in water. When the wood is old or unusually hard, the maceration must be prolonged or the 
specimen may be boiled for a short time. Longitudinal sections may be cut by gluing the piece of stem to a cork to afford a hold upon it.

The preparation of sections of minerals and rocks is usually considered a very difficult matter, but much may be accomplished without the aid of the usual lapidaries' wheel for cutting and the revolving lap for grinding the sections, if the microscopist provides himself with a flat piece of lead six to ten inches square, and two pieces of boiler plate of the same size, planed on one side. A chip of the rock may be ground flat on the leaden lap, charged with coarse emery and water, and the process continued with emery of moderate grain on one of the iron plates, and the finest flour emery on the other. The flat side being then cemented with balsam (undiluted) to a piese of plate-glass about an inch square, the process of grinding may be repeated on the other side of the chip, until it becomes perfectly transfarent. It may then be detached from the glass by soa':ing in benzole, and mounted in balsam in the usual way.

When the sections are to be mounted dry, which is very rarely the case, the fine scratches left by the flour emery must be removed by giving the section a final polish on a hard and flat oilstone reserved for the purpose and wetted with clean water only.

When it is desired to preserve the natural colour of objects, espe ially of such as contain chlorophyll, the necessary preliminary treatment with alcohol raises an objection to the balsam process, and another objection is that some tissues are rendered too transparent, and many of their finer features are obliterated by the highly refractive balsam.

In these cases the object must be mounted in some aqueous medium, the best and most convenient being a preparation of glycerine and gelatine, which forms a transparent jelly when cold, but is easily liquefied by heat. It is best to buy this "glycerine jelly," as it is troublesome to make on a small scale. When required for use it must be liquefied by standing it in a cup of hot water.

In general, objects to be mounted in glycerine jelly should not be embedded, but if any support is needed in cutting, this should ta'se the form of two pieces of cork hollowed out to the shape of the object.

Water in the objects no longer presents any difficulty in this method of mounting, but air has still to be contended with, and the methods adopted for its elimination in the balsam process are no longer applicable. Some objects may be freed from air by boiling in water for a few minutes, but many would be spoilt by such treatment. Recourse must then be had to the air-pump, or, if this instrument is not accessible, to a very simple process depending on the great solubility of air in water. ${ }^{1}$

A wide-mouthed bottle of about four ounces capacity, with a closely fitting solid stopper, is completely filled with water, which at the time is, and for half an hour previously has been, boiling, in order to expel all traces of dissolved air. The stopper being then inserted without inclosing a single air-bubble, the bottle is set aside until cool enough to receive the sections, which are then to be put into it. A few drops of boiling water are then to be added to make good the inevitable loss in removing the stopper; the bottle is to be again closed, wiped dry, and securely sealed with melted paraffin. After twelve hours it may be opened, and the whole contents turned into a white forcelain shallow dish. The sections can then be easily seen, and picked out with a section-lifter, and should be soaked for half an hour in a 50 per cent. solution of glycerine before mounting.

The process of tran-ferring the object to the slide, applying the liquefied jelly, and lowering the cover, are exactly the same as in the balsam method, and the slide should be set aside in a clip for a few hours for the jelly

I The writer cannot remember where be has seen this process described, but he can testify to its efficiency. to solidify. In cold weather it is advisable to warm the brass table by means of a spirit lamp, or the jelly may viscify too quickly.

When quite cold and set, the excess of jelly may be cleaned from the edges of the cover glass, and the slide may then be ringed with asphalte while running in the turntable. Two or three subsequent coats of asphaite and the attachment of labels will complete the slide.

The objects for which glscerine jelly is most suitable are the lower forms of vegetable life--Algæ, Desmidiaceæ, Characeæ, Hepaticæ, Fungi, Lichens, Mosses, \&c., and cuticles and sections of plants of all kinds. Many animal tissues are also better seen in it than in balsam.

It is but seldom that other preservative media are required, and it will be found that almost all objects may be suitably preserved by one of the three methods here described.

Closely related to the preparation of objects for microscopic examination is their delineation by photography, an art of the greatest value on account of its freedom from bias and personal equation, and as a means of lecture illustration with the aid of the lantern it must be appreciated by the number; who have experienced the difficulty of demonstrating microscopic structure to many persons.

This application of photography, which is almost as old as the photographic art itself, extending back to the days of Daguerreotype, owes its recent development and simplification mainly to the introduction of gelatine plates, and the object of Mr. Malley's work ${ }^{1}$ (which, however, should be called Photomicrography, for it does not treat of the production of microscopic photographs, as its name would imply) is to show how in an ordinary room, with an ordinary microscope, photographic camera, and paraffin lamp, photographs can be taken which will bear comparison with those obtained in the old days by the aid of sunlight reflected from expensive heliostats, electric arcs, magnesium and lime light, microscopes of special construction, and rooms specially set apart for the work. It therefore appeals to a large class of personsthose who would wish to practice the art, but lack either the sunlight hours or the expensive illuminators and apparatus formerly considered necessary.

The microscope, camera, and dark room, with their accessories, and the method of working with the Swan incandescent lamp and sunlight are described in detail, but the reader is perplexed by references to an illustration which cannot be found in the book. Four Woodburytype reproductions of photomicrographs of Aulaucodiscus, Pleurosigma, and Surirella, scales of Lepisma, and Bacilli in human lung, accompany the work.

The instructions for taking negatives by the wet collodion and gelatino-bromide processes and the production of positives, enlargements, \&c., are clear and concise, but we must enter an emphatic protest against the author's opinion that in object-glasses for photomicrography, depth of focus or penetratic $n$ is to be sacrificed to angular aperture. Penetration and flatness of field are really of greater importance in lenses for photographic than for visual purposes, for in viewing an object under the microscope the observer bas the power of focusing in rapid succession, and by imperceptible gradations, points at different depths and different distances from the centre of the field; but a photograph represents only such structures as were in focus at the time of exposure, and once taken, the focus is unalterable. It is therefore desirable to secure as great a depth of focus and as flat a field as possible-qualities which are incompatible with large apertures.

Mr. Malley very properly advises his readers not to walk about during the exposure of a plate, but the extension of the prohibition to speaking also is surely an unnecessary restraint.

I "Micro-Photography," by A. Cowley Malley, B.A., M.B., \&c. (Lewis, Gower Street.) 